\title{
A Novel Linear Air Amplifier Technology to Replace Rotary Fans in Data Center Server Rack Cooling
}

\author{
Sajad Alimohammadi ${ }^{1,2,}$, Tim Persoons ${ }^{2}$ \\ ${ }^{1}$ School of Mechanical and Design Engineering, Technological University Dublin, City Campus, Ireland \\ ${ }^{2}$ Department of Mechanical and Manufacturing Engineering, Trinity College Dublin, Dublin, Ireland \\ * Corresponding Author: sajad.alimohammadi@tudublin.ie, +35314023900
}

\begin{abstract}
This paper investigates the potential of a novel linear air flow amplifier designed to replace rotary fans for various industries where their performance is undesirable like electronics cooling applications in compact data center servers. The device operates with no moving parts and generates a high-speed uniform flow attached to the rack server surface with a need for a detailed investigation into the mechanism of fluid flow, heat transfer, and noise generation associated with its operation. To progress the state of the art, a high aspect ratio rectangular air amplifier is developed and characterized from various engineering aspects to address the flow performance, energy efficiency and acoustic performance of the new devices in a side by side comparison versus high-speed axial fans. As the preliminary effort in the development of the novel air amplifier, this paper aims to investigate the hydrodynamic characteristics of the air amplifier using analytical and experimental methods.
\end{abstract}

\section{Introduction}

Data centers and telecommunication systems are critical components of every modern society, with a growth of $15 \%$ in the business of data centers in a four-year period before 2019 [1]. Technologies like AI, IoT and 5G will only further contribute to increasing the demand for DCs [2].

Like most of the electronics, DCs still heavily depends on aircooling systems to maintain the continuous operation of the server racks. In air-cooled data centers with fans as the air movers, currently, between $15-40 \%$ of the total power consumption is used only to run the electronics cooling system [3]. Air cooling is favored due to its accessible nature, easier maintenance, and the air itself as the cooling medium not posing potential. The operation of the fans, however, involves some of the key challenges in data center thermal management. A couple of adverse effects associated with fans in the cooling systems can be listed as low cooling efficiency, high noise levels, and operational and maintenance costs.

\section{Air Amplifier}

Air amplifiers are designed to utilize the Coandă effect [4] by increasing the velocity of air flow ejected as compressed air (jet) to surrounding air, inducing secondary flow entrainment. Various industries are involved in the manufacturing of commercial annular air amplifiers for different applications in cooling or pumped flow multiplication such as Dyson Air Multiplier ${ }^{\mathrm{TM}}$ technology [5] and Brauer Air Amplifiers [6].

Figure 1 displays a schematic diagram of the novel linear air amplifier designed in this study, with the primary flow and entrained (secondary) flow depicted, installed flush with data center server rack inlet section. This device is fundamentally designed by tailoring the existing annular air amplifier technology to suit the server cooling applications.

The linear air (flow) amplifier, similar to annular designs, relies on entrainment of surrounding air due to a high-velocity air jet, typically using a continuous Coandă wall-bounded flow. This device has the potential to achieve reasonable hydrodynamic characteristics, high heat transfer rates, and lower noise compared to equivalent axial fans.

Scaling laws to define various operational characteristics for axial fans are proposed in reference handbooks [7]. There is little to no information on the scaling laws for a device such as the linear air amplifier. The first stage of this study presents the results for analytical and experimental investigations on characterizing the primary flow rate and amplification (entrainment) performance of the wall-bounded jet emerging from the air amplifier.

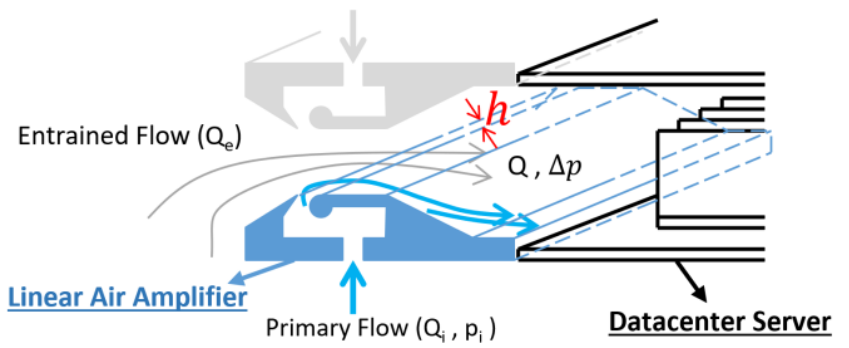

Figure 1: schematic diagram of the novel air amplifier

\section{Experimental Methodology}

For the measurements, a modular experimental test facility has been developed for objectively comparing various operational metrics of axial fans used in servers (Nidec UltraFlo $^{\mathrm{TM}}$ family from reference server: Intel S2600TP), commercial annular air flow amplifiers (Brauer Fixed Gap 
Airmover ${ }^{\mathrm{TM}}$ ) and the in-house designed air flow amplifier (schematically displayed in Fig. 2 (a)). The displayed version of the linear air amplifier is composed of 3 separate pieces, namely, left block and the stator diffuser printed in a single piece, right block with matching groove to fit with the diffuser, and moveable nozzle. Primary air nozzle gap width, $h$, is adjustable using a micrometer translation stage which controls the movable nozzle section motion in line with the diffuser block.

2 (b) displays the apparatus designed to measure volumetric flow rate, pressure head, heat transfer, and sound power induced by operation of both axial fans and the linear air flow amplifier as exchangeable equipment in this modular setup.

The experimental setup is composed of a flow channel, 40 by 150 by $500 \mathrm{~mm}^{3}$, corresponding to the selected reference

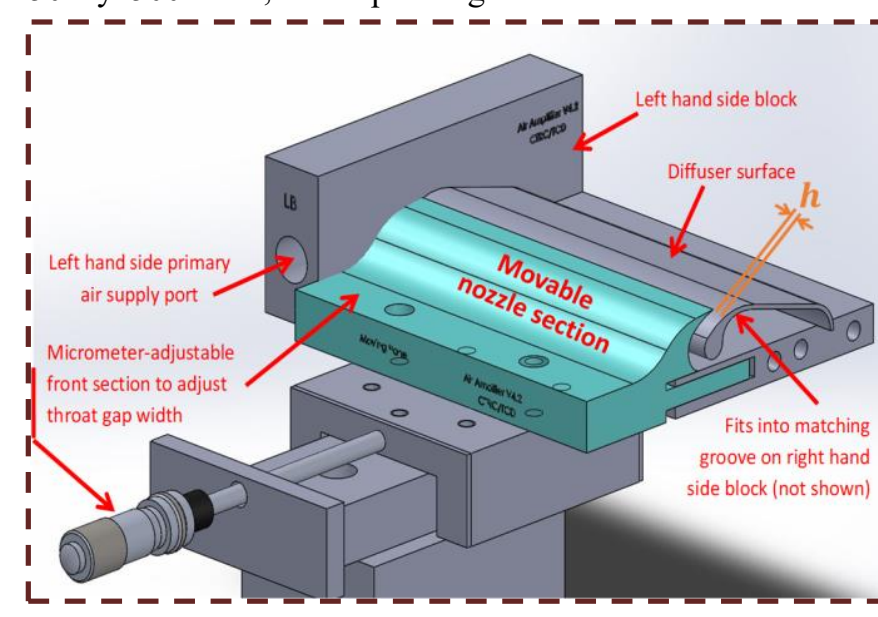

(a)

blade server: Intel S2600TP, with a heated metal foil using copper electrodes at the bottom.

Three parallel axial fans or the new air flow amplifier can be installed at the inlet of the duct providing air cooling requirements similar to the blade server cooling system. IR camera can record temperature distribution over the foil from the bottom.

Finally, six removable perforated plates are used to enable the controllability of the backpressure during the measurements.

This study only reports the results for the flow rate and pressure head measurements carried out for both air flow amplifier and axial fans.

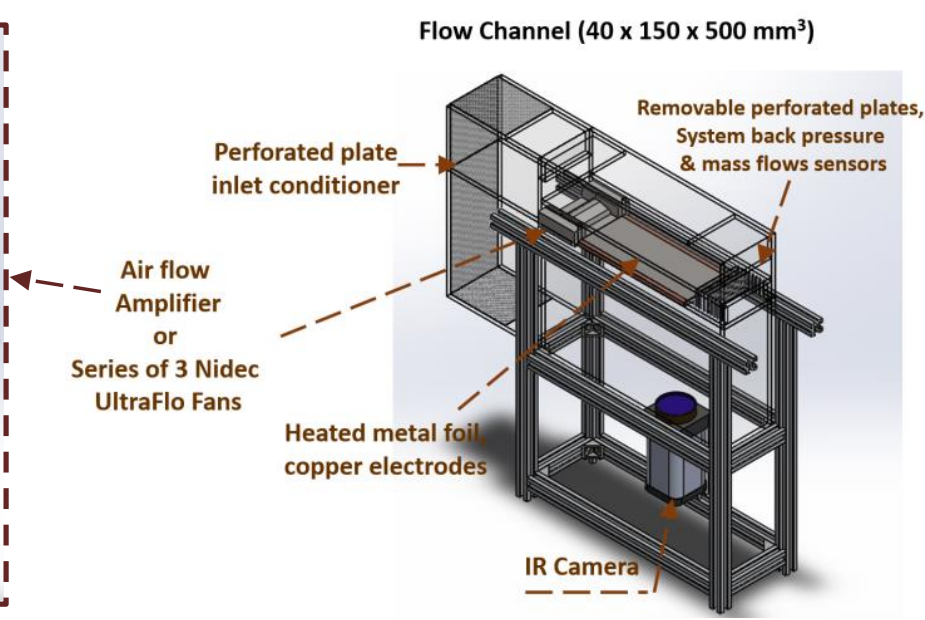

(b)

Figure 2: a) 3D printed air flow amplifier made of 3 blocks, b) schematic diagram of experimental measurement apparatus

\subsection{Scaling laws for axial fans}

Based on scaling laws of axial fans for volumetric flow rate $Q$ and pressure head $\Delta p[7]$ and assuming that the pressure head varies quadratically with flow rate, the following can be derived:

$$
\begin{gathered}
Q=c_{Q}\left(\frac{n}{60}\right)\left(\frac{d}{1000}\right)^{3} 60\left[\mathrm{~m}^{3} / \mathrm{min}\right] \\
\Delta p=c_{\Delta p} \rho\left(\frac{n}{60}\right)^{2}\left(\frac{d}{1000}\right)^{2}\left(\frac{L}{d}\right)^{1 / 2}[\mathrm{~Pa}]
\end{gathered}
$$

where $n$ is the rotational speed [rpm], $d$ is the impeller diameter [mm] and $L$ is the fan length [mm], $\rho$ is the air density, and $c_{Q}$ and $c_{\Delta p}$ are specific fitting factors to be derived based on a wide selection of axial fans used in the selected server.

The above can be utilized to predict actual values, i.e. the pressure head vs flow rate relation or PQ curve, for the fans under different operating conditions and dimensions, i.e. the rotational speed $n$ and impeller size $d$. The results are discussed in section 4.1 .

The reference quantities are lumped together in specific fitting factors $c_{Q}$ and $c_{\Delta p}$. Using the database generated from 39 fans from the Nidec UltraFlo family, correlations are fitted giving values for $c_{Q}$ and $c_{\Delta p}$ detailed below:

$$
\begin{aligned}
& c_{Q}=0.348 \pm 0.07\left(R_{Q}^{2}=0.985\right) \\
& c_{\Delta p}=2.264 \pm 0.34\left(R_{\Delta p}^{2}=0.982\right)
\end{aligned}
$$

Reference coefficients above are specific for the aforementioned family of fans.

\subsection{Scaling laws for linear air amplifier}

The same approach as section 3.1 can be applied to relate the air amplifier flow performance to operating parameters. This involves developing scaling laws for the linear air amplifier designed in this research with specific fitting factors derived using fitted empirical correlations.

For this purpose, a set of experiments are carried out for different operating conditions and dimensions on the air 
amplifier. This involves varying primary jet gap width $h$ under variable system backpressures $\Delta p$ and monitoring primary jet flow rate $Q_{i}$, total flow rate $Q$, and pressure head $\Delta p$ as measured quantities. The results are discussed in section 4.2 .

\subsection{Particle image velocimetry}

Particle Image Velocimetry PIV measurements are carried out at the entrance region to the channel with the focus on the air amplifier to help visualize the inherent flow physics taking place during the operation of the device. This is mainly used to assess the effect of backpressure on the entrained flow and to closely highlight the Coandă wall-bounded flow phenomenon.

A CMOS camera is used in an angled arrangement (LaVision High-Speed Star 6 at 500 fps with $1024 \times 1024$ px $^{2}, 12$ bit). Seeding is glycol/water aerosol, with a particle diameter of $0.2-0.3 \mu \mathrm{m}$. It is essential to entrain to be injected in the primary supply of the device and to be ejected through the gap width of the amplifier. For this purpose, a venturi ejector fabricated for this purpose is designed and 3D printed which is installed at the high-pressure inlet of the air amplifier. The beam of a Nd:YLF twin cavity laser (up to $15 \mathrm{~mJ}$ per pulse at $1 \mathrm{kHz}$ ) passes through customized optics to form a fine lightsheet aligned to the mid-plane of the air amplifier(see Fig. 3).

LaVision DaVis 7.2 software was used for image capturing and post-processing of the velocity fields, using multi-grid cross-correlation with continuous window shifting and deformation [8].

The prototype air amplifier is 3D printed from fluorescent orange PA to avoid excessive surface reflections due to 527 $\mathrm{nm}$ laser sheet. With the camera facing the prototype from the side, the right-hand side block of the air amplifier is redesigned to provide better optical access (see Fig. 3).

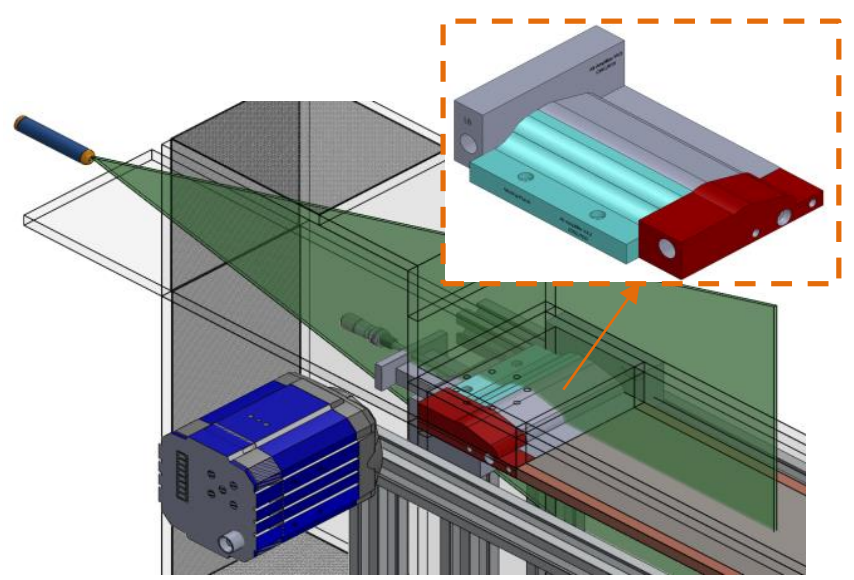

Figure 3:schematic of particle image velocity PIV setup

\section{Results}

The results for the experimental and numerical study of the flow performance of both axial fan and the in-house designed air flow amplifier with rectangular jet orifice are reported in this section.

\subsection{Fan Performance Analysis}

The fitted empirical correlations described as scaling laws for Intel server S2600TP axial fans in section 3.1 are used to develop a prediction tool to estimate the PQ curves for fans. This is done by changing the total flow rate moved by a series of 3 axial fans (corresponding to a half-width blade server) using the backpressure induced by incorporating perforated plate inserts from 1 to 6 . Figure 4 displays the results with the fan operating at the maximum speed of $21,400 \mathrm{rpm}$.

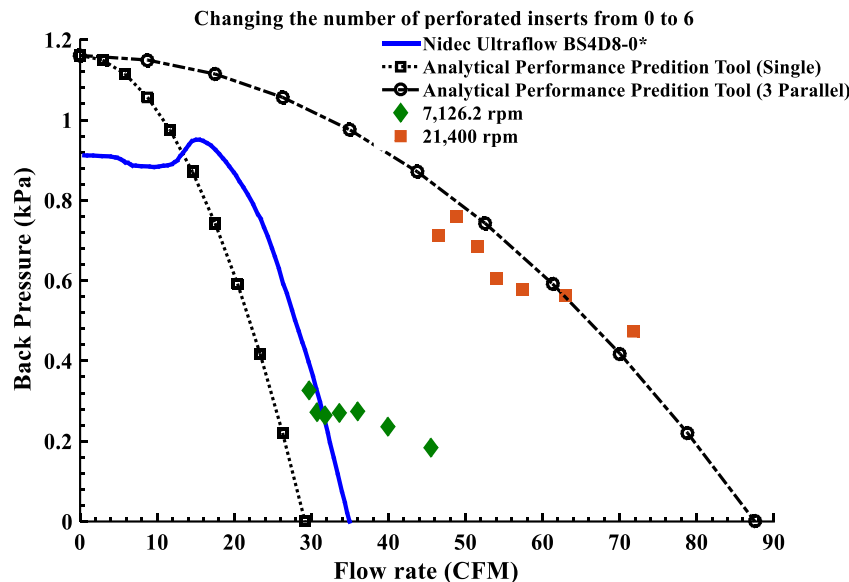

Figure 4: comparison of $P Q$ curves for Intel server S2600TP axial fans using predicted values (Eqs. 1-4), measured quantities from the experiments, and manufacturer's database (Nidec UltraFlo ${ }^{\mathrm{TM}}$ family)

\subsection{Air amplifier characterization}

The variation of the primary jet flow rate $Q_{i}$ as a function of the primary jet gap width $h$ in the presence of an adjustable inlet pressure $p_{i}$ is reported in Fig. Figure 5.

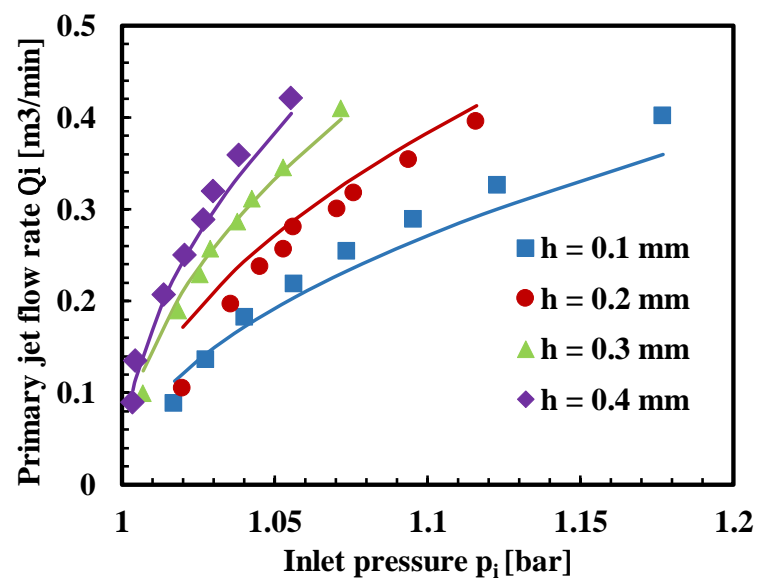

Figure 5: experimental results (markers) and fitted correlations (solid lines) for variation of primary jet flow rate $\left(Q_{i}\right)$ with adjustable jet gap width $(h)$ and primary inlet pressure $\left(p_{i}\right)$ 
Fitted empirical correlations based on compressible flow theory through variable restrictions [9] are generated to relate the air amplifier flow performance to operating parameters such as inlet pressure and jet gap width:

$$
Q_{i}=c_{d} A_{t} \frac{p_{i}}{\sqrt{R T_{i}}} S
$$

where the least square fitted discharge coefficient is found as:

$$
c_{d}=3.22\left(\frac{h}{0.1}\right)^{-0.50}, R^{2}=0.975,3.9 \% \text { rms deviation }
$$

and,

$$
S= \begin{cases}\left(\frac{p_{i}}{p_{a}}\right)^{-\frac{1}{\kappa}} \sqrt{\frac{2 \kappa}{\kappa-1}\left(1-\left(\frac{p_{a}}{p_{i}}\right)^{\frac{\kappa-1}{\kappa}}\right)} & \text { if } \frac{p_{i}}{p_{a}} \leq\left(\frac{\kappa+1}{2}\right)^{\frac{\kappa}{\kappa-1}} \text { (a) } \\ \sqrt{\kappa\left(\frac{2}{\kappa+1}\right)^{\frac{\kappa+1}{\kappa-1}}} & \text { if } \frac{p_{i}}{p_{a}}>\left(\frac{\kappa+1}{2}\right)^{\frac{\kappa}{\kappa-1}} \text { (b) }\end{cases}
$$

Condition (a) above refers to subsonic fluid flow and condition (b) refers to a choked flow condition.

Figure 6 displays the flow rate amplification, or total to primary flow rate, $Q / Q_{i}$, under varying jet gap width $h$ in the range of $0.1 \mathrm{~mm}$ to $0.3 \mathrm{~mm}$ and in the presence of an adjustable inlet pressure $p_{i}$.

The results indicate that almost at all inlet pressures, the flow rate amplification factor for the air amplifier remains highest at jet gap width of $0.1 \mathrm{~mm}$.

Fitted correlation for the flow rate amplification $Q / Q_{i}$ induced by the operation of the air amplifier as a function of gap width $h$, primary flow rate $Q_{i}$, and system backpressure $\Delta p$ can be derived as below:

$$
Q=a Q_{i}^{m+1}\left(1-\frac{\Delta p}{b Q_{i}^{n}}\right) A_{t}^{q}
$$

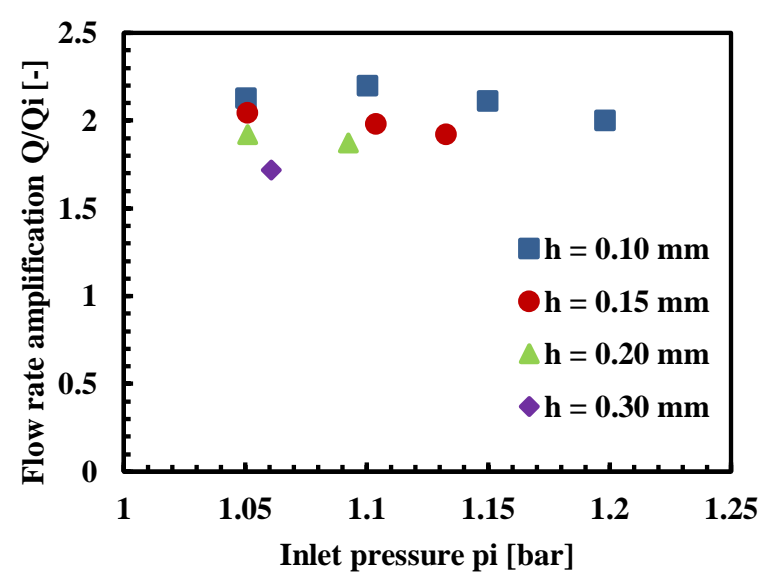

Figure 6: effect of inlet pressure $p_{i}$ and jet gap width $h$ on flow amplification $Q / Q_{i}$ where, the above, with $Q_{i}$ from compressible flow theory through variable restrictions, Eq. (5), can be rearranged as:

$$
\frac{Q}{Q_{i}}=a Q_{i}^{m}\left(1-\frac{\Delta p}{b Q_{i}^{n}}\right) A_{t}^{q}
$$

Varying perforated plate inserts, from 0 to 6 , at the end of the Perspex measurement channel (half-width blade server) offers adjustable primary inlet pressure $\left(p_{i}\right)$ and provides the flow rate vs backpressure data points for the in-house design air amplifier as displayed in Fig. 7.

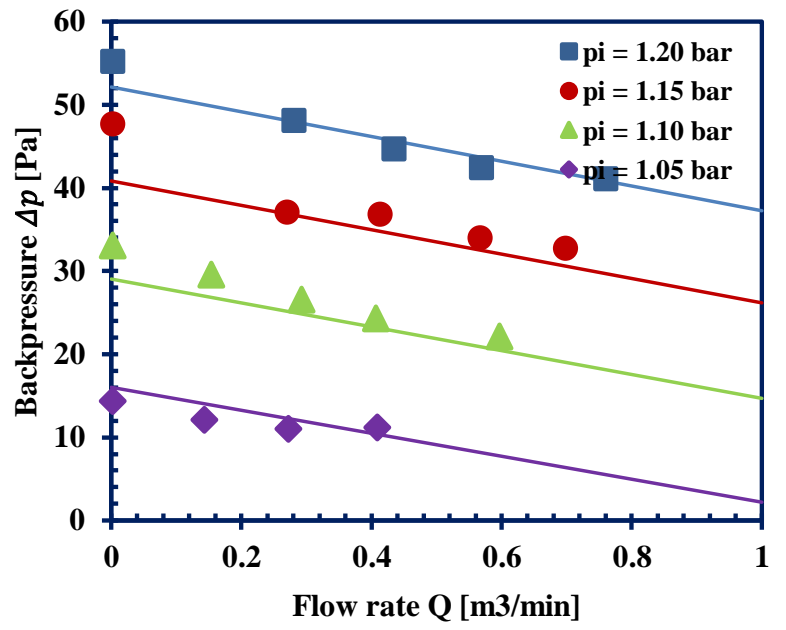

Figure 7: $P Q$ curve for air amplifier including experimental results (markers) and fitted correlations (solid lines)

Treating the backpressure $\Delta p$ vs flowrate $Q$ relation for the air amplifier as a fan curve helps to correlate the data in Fig. 7. into the following expression:

$$
\Delta p=b Q_{i}^{n}\left(1-\frac{Q}{a Q_{i}^{m+1} A_{t}^{q}}\right)
$$

where, least squares fitted parameters are as $a=225, b=$ $270, m=0.61, n=-1.09, q=1.72$ with $R^{2}=0.772$, $14.5 \%$ rms deviation for all fitted parameters.

The results for air amplifier PQ curve indicate a linear relationship between flow rate and backpressure where the maximum flowrate almost doubles by increasing the inlet pressure from 1.05 bar to 1.2 bar.

\subsection{PIV flow field analysis}

Figure 8 displays PIV measurement results to visualize the air amplifier flow field at the maximum flow rate (minimum backpressure; left-hand side) and zero flow rate (maximum backpressure; right-hand side). This can be used to further investigate the flow features and physics associated with the performance of the air amplifier as displayed on the figure. This also helps to perform an analysis of mechanisms of flow entrainment, wall attached flow and turbulent jet ejection into the surrounding air. 

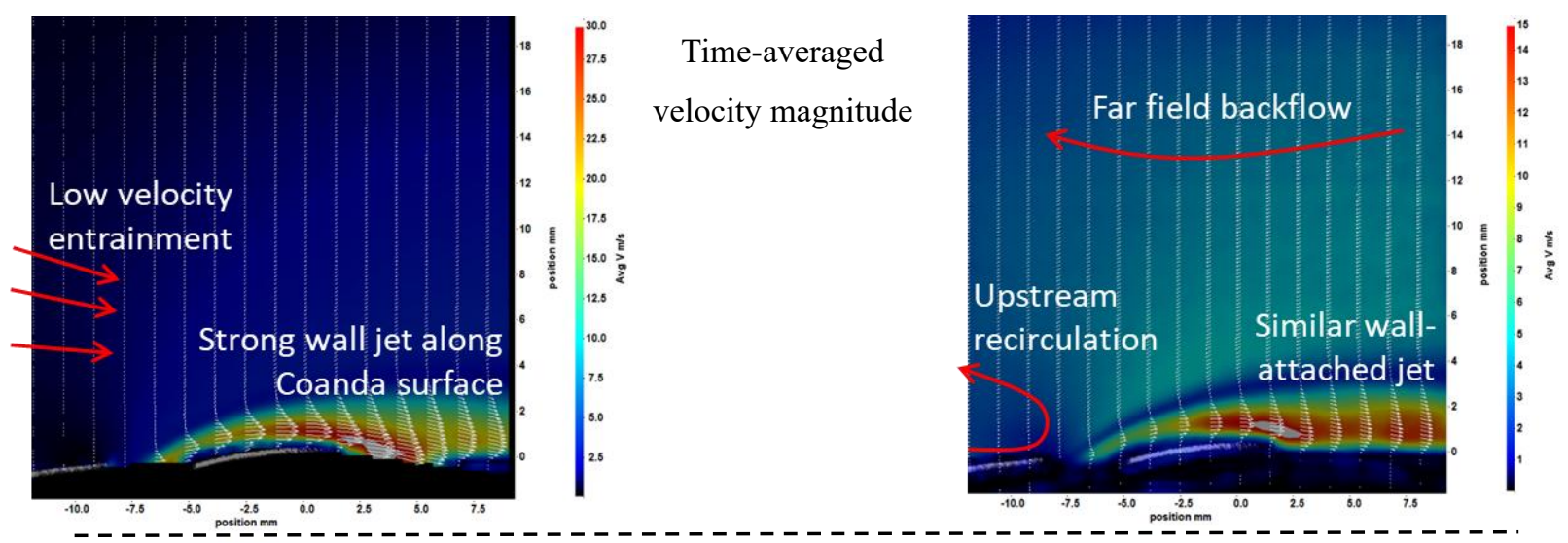

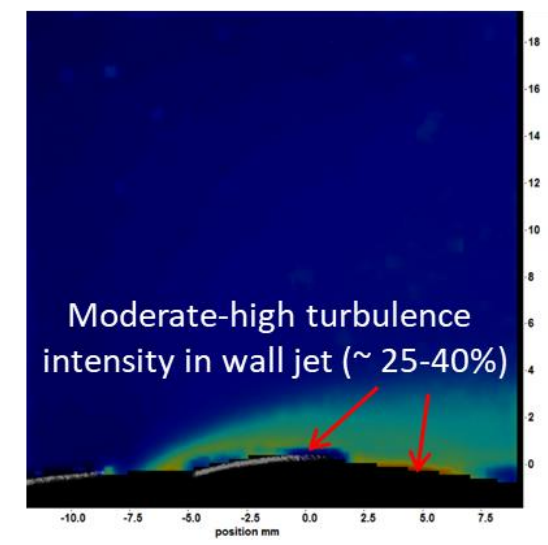

a) Max. flow, min. backpressure

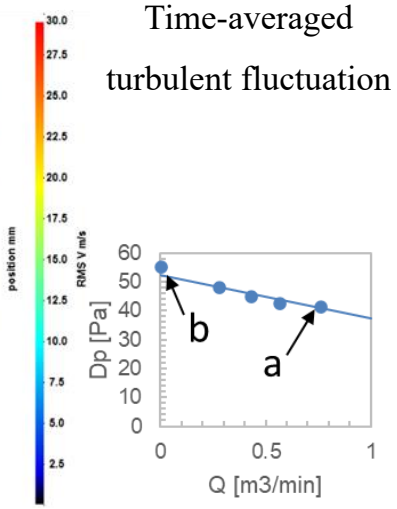

b) Zero flow, max. backpressure

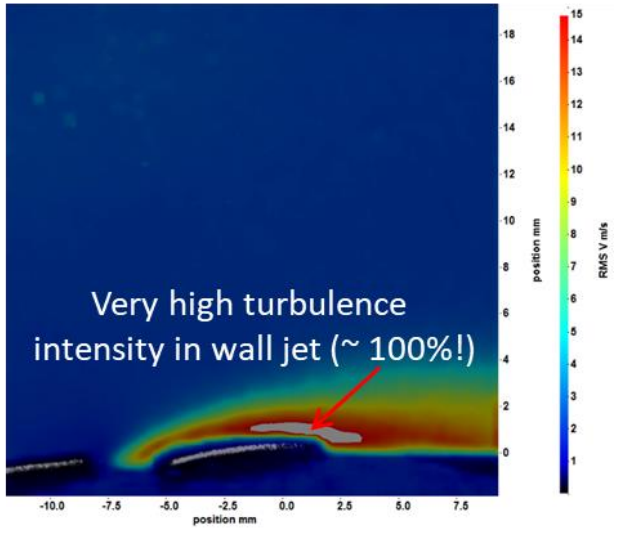

Figure 8:PIV flow filed analysis for time averaged velocity magnitude (top) and turbulent fluctuation (bottom)

\subsection{Discussion: $P Q$ curve for air amplifier vs axial fans}

For variation of primary jet flow rate $(Q)$ vs backpressure $\Delta p$, the fan curve or PQ curve for both air amplifier and series of three axial fans at the measurement channel inlet is shown in Fig. 9. This is done with adjustable primary inlet pressure $\left(p_{i}\right)$ for air flow amplifier (using perforated plate inserts) and adjustable rotational speed for fans.

The below is an overall insight into the side by side comparison between the traditional device (fan) vs the novel device proposed in this research when it comes to air moving capacity.

In summary, when comparing air amplifier performance to series of Nidec fans used in Intel S2600TP server:

- For setting with same max flow rate, fans deliver higher $\Delta p$

- $\Delta p=f(Q)$ for fans is quadratic vs linear for air amplifier

- However, air amplifier at $h=0.1 \mathrm{~mm}, p_{i}=1.45 \operatorname{bar}(\boldsymbol{\square})$ performs similarly to fans at $33 \%$ speed $(\square)$

The overall results for side by side analysis of fan series performance vs the novel air amplifier can be perfectly described using the scaling laws, described earlier in this report. This can be used to develop a prediction tool to estimate flow rate and pressure head delivered by both devices, i.e. PQ curves.

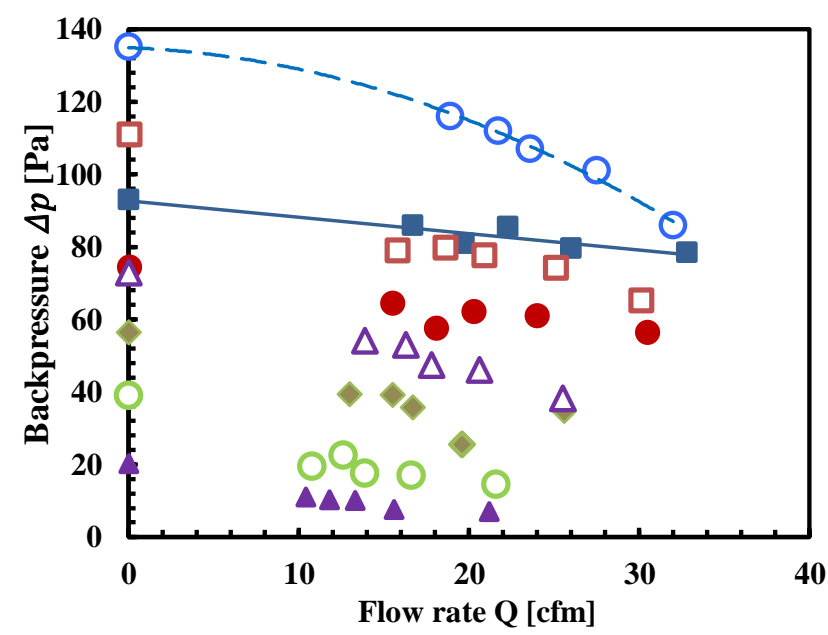

$\square$ pi $=1.45$ bar $\bigcirc$ pi $=1.33$ bar $\diamond$ pi $=1.20$ bar $\Delta$ pi $=1.12$ bar O Fans at $35 \%$ Fans at $33 \% \Delta$ Fans at $28 \%$ Fans at $24 \%$

Figure 9: experimental result for PQ curves: series of axial fan (hollow markers) vs air amplifier (filled markers) 

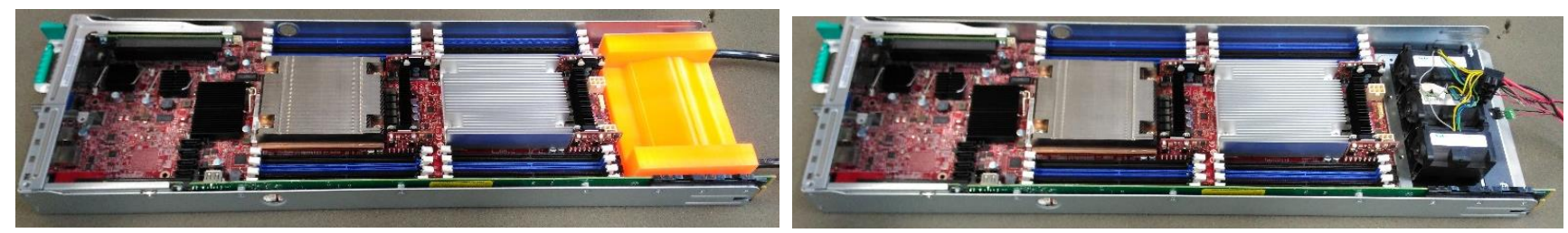

Figure 10: Lab demonstrator of air amplifier (left) vs fan series installed in the reference Intel S2600TP server

Figure 10 displays the lab demonstrator prototype of the air amplifier (printed in fluorescent orange PLA for PIV) in comparison with fan series installed in the reference server.

A summary of the relations described using the scaling laws for fans and air amplifiers, Eqs. (1) - (8), can be listed as Table 1.

Table 1: parametric relations based on scaling laws for fans vs air amplifier to estimate volumetric flow rate $Q\left(\mathrm{~m}^{3} / \mathrm{min}\right)$ and pressure head $\Delta p(\mathrm{~Pa})$

\begin{tabular}{l|c|c} 
& Axial fans & Air amplifiers \\
\hline Inputs & $\begin{array}{c}\text { Angular speed } n \\
\text { Impeller size }\end{array}$ & $\begin{array}{c}\text { Inlet pressure } p_{i} \\
\text { Gap width } h \text {, span l }\end{array}$ \\
\hline Flow rate & $Q \propto n d^{3}$ & $Q_{i}=f_{1}\left(p_{i}, h\right)$ \\
\hline Pressure & $\Delta p \propto n^{2} d^{2}$ & $Q=f_{2}\left(\Delta p, Q_{i}\right)$ \\
\hline
\end{tabular}

\section{Conclusions}

This a research into hydrodynamics characterization and the flow performance of a novel linear air amplifier to improve the reliability of traditional air-cooled data center servers. The device operates by emitting a high-speed uniform flow attached to the rack server surface with a need for detailed investigation associated with the operation of the linear air amplifier.

This stage of the research determines the potential of air flow amplifiers to rival and replace axial fans in terms of achievable flow rate and amplification at given backpressure. Analytical models and correlations of the newly designed device are fitted to the measured behavior of both axial fans and the novel air flow amplifier. This helps to investigate the agreement between analytical and experimental results.

The device also offers the potential for significant improvements in surface heat transfer given the wall-bounded nature of the high-speed, amplified flow. However, the total energy consumption for the new air amplifier in comparison to traditional fans would be the key outcome of these preliminary investigations in order to reflect on the feasibility of air amplifier as a replacement for traditional fans in data center server cooling system.

\section{Acknowledgements}

The authors acknowledge the financial support of the NSF I/UCRC Cooling Technologies Research Center (CTRC) under project 'Low Noise Air Amplifiers'. The authors acknowledge the support of the DJEI/DES/SFI/HEA Irish Centre for High-End Computing (ICHEC) for the provision of computational facilities and support. The authors also acknowledge the financial support of the Irish Research Council (IRC) under grant number GOIPD/2016/216

\section{Literature}

[1] S. V. Garimella, T. Persoons, J. A. Weibel, and V. Gektin, "Electronics Thermal Management in Information and Communications Technologies: Challenges and Future Directions", IEEE Trans. Compon. Packag. Manuf. Technol., vol. 7, no. 8, pp. 1191-1205, Aug. 2017.

[2] L. Gibbons, D. Kennedy, B. Coyne, E. Denny, E., S., Alimohammadi, "A Techno-Economic Analysis of Current Cooling Technologies within Irish Data Centres", IEEE 25th Int. Worksh. Therm. Invest. ICs and Syst. (Therminic), Italy, Sep 2019.

[3] A. C. Kheirabadi, D. Groulx, "Cooling of Server Electronics: A Design Review of Existing Technology", Appl. Therm. Eng., 105, pp. 622-638, 2016.

[4] H. Coanda, "Device for Deflecting a Stream of Elastic Fluid Projected into an Elastic Fluid". United States of America Patent 2,052,869, 1936

[5] Dyson, "Dyson Cool ${ }^{\mathrm{TM}}$ fans", [Online]. Available: https://www.dyson.co.uk/fans-and-heaters/dyson-cooltechnology.html. [Accessed 1 Aug 2020].

[6] Brauer, "Standard Fixed Gap Air Amplifier", https://www.brauer.co.uk/standard-fixed-gaps386.aspx. [Accessed 1 Aug 2020].

[7] C. M. Harris (Ed.), Handbook of Acoustical Measurements and Noise Control, 3rd Ed., McGrawHill: New York, 1991

[8] S. Alimohammadi, J. McEvoy, Yan Delauré, T. Persoons, "Benchmarking the Application of Detached Eddy Simulation Techniques in Data Center Server Flow Modelling using Stereoscopic Particle Image Velocimetry", IEEE 24th Int. Worksh. Therm. Invest. ICs and Syst. (Therminic), Sweden, Sep 2018.

[9] F. M. White, Fluid Mechanics, 7th Ed., McGraw-Hill: New York, 2011. 